Digital Press Social Sciences and Humanities

Mise en place de l'apprentissage intégratif des quatre compétences langagières au but de renforcer l'autonomie d'apprenant

Herman

Proceeding of Conférence internationale sur le français 2018

Joesana Tjahjani, Merry Andriani, Sajarwa, Wening Udasmoro (eds) 


\title{
Mise en place de l'apprentissage intégratif des quatre compétences langagières au but de renforcer l'autonomie d'apprenant
}

\author{
Herman \\ Universitas Negeri Yogyakarta, Yogyakarta, Indonesia \\ e-mail : herman@uny.ac.id
}

\section{Résumé}

Le but de cet article est de proposer, comme sujet de discussion, un modèle d'apprentissage du français langue étrangère qui intègre les quatre compétences langagières : La production orale, la production écrite, la compréhension orale et la compréhension écrite ayant des fins de l'autonomie d'apprenant. La recherche a été mise en place chez les étudiants de cinquième semestre du mois d'octobre au mois de décembre 2017, au département du français de l'UNY qui réunit les cours des quatre compétences. La recherche s'appuie sur la théorie d'apprentissage coopératif et la mise en place des stratégies d'apprentissage de langue. Elle montre que l'apprentissage intégratif permet aux élèves d'avoir une toile de fond plus proportionnelle où les enseignants et les apprenants établissent librement des contacts en utilisant les langues cibles sans être contraints par leurs différents intérêts. L'apprentissage implique également sur l'importance de la facilitation et de la création d'une occasion (affordance-angl.) qui dispose un milieu pour s'immerger dans la langue cible.

\section{Mots-clés}

apprentissage intégratif, les stratégies d'apprentissage, l'autonomie d'apprenant

\begin{abstract}
This article is aimed to propose a model of learning of French as foreign language which integrates the four language skills: speaking, written production, listening comprehension and reading comprehension with purposes of autonomy of learner. The research has been implemented in the fifth semester students from October to December 2017, in the French Department of UNY which brings together courses of those four skills. The research relies on the cooperative learning theory and the theory of language learning strategies. It shows that the integrative learning allows students to have a more proportional background where teachers and learners could freely establish contacts in the languages targets without being constrained by their different interests. Learning involves also on the importance of facilitating and creating an affordance, which has an environment to immerse themselves in the target language.
\end{abstract}

\section{Keywords}

integrative learning, the strategies of learning, autonomy of learner

\section{Introduction}

Rubin (1975) dans un article intitulé « ce que le bon apprenant peut nous apprendre >> présente l'attitude, le comportement positif des apprenants en langues vis-à-vis de la langue cible comme facteur susceptible qui permettent à certains d'entre eux d'atteindre la réussite. L'attitude ou le comportement évoqué par Rubin est connu sous le nom de «la stratégie d'apprentissage des langues » (Rubin, 1975, p. 42). Le terme 'bon apprenant' de langue désigne les apprenants qui développent leurs stratégies d'apprentissage pour les rendre capable à surmonter de diverses difficultés d'apprentissage. Selon Rubin, 
à part de plusieurs autres facteurs affectifs, l'importance porte sur la possibilité de ces bons comportements qui puissent être enseignés aux autres apprenants 'faibles'. Plus tard, cette stratégie d'apprentissage des langues est devenue très intéressante pour les didacticiens des langues, en particulier ceux qui sont liés aux effets sur le processus et à la réussite de l'apprentissage des langues comme facteur dérivé des apprenants eux-mêmes.

Oxford (1990) distingue les stratégies d'apprentissage des langues en deux types, à savoir les stratégies directes, y compris trois sous-stratégies : les méthodes mnémoniques, s. cognitives et s. compensatoires, et les stratégies indirectes qui comprennent trois sous stratégies: métacognitives, affectives et sociales. On trouvera plus de détails sur chacune de ces stratégies dans plusieurs autres références qui renvoient également à des typologies produites par Oxford (1990), Rubin (1989) O'Malley et Chamot (1990). Dans le même article, en plus d'initier des stratégies d'apprentissage, Rubin a proposé trois variables fortement liées à la réussite d'apprenant, d'abord l'aptitude, la motivation et les opportunités. Parmi les trois, les opportunités et les motivations, ont été plus tard évoquées par Oxford (2003) en deux thèmes sous l'angle de l'autonomie de l'apprentissage des langues. Les variables motivationnelles sont reconnues depuis longtemps comme un facteur déterminant du succès de l'apprentissage des langues, mais la variable « opportunité » requiert un fondement théorique assez convaincant jusqu'à ce qu'Oxford le considère comme "ce qui est abordable" pour assurer le processus d'apprentissage des langues. Les stratégies d'apprentissage restent la clé principale pour favoriser l'autonomie d'apprenant d'une langue. Mais l'absence de "ce qui est abordable" pour les apprenants affaiblira ou empêchera leur créativité et les éloignera de leurs propres forces.

L'autonomie de l'apprenant peut être considérée comme une responsabilité pour déterminer l'autoefficacité jouée par les apprenants français. Le rôle qui était précédemment déterminé par les enseignants est maintenant divisé proportionnellement. Cela soulève plusieurs questions sur le modèle d'apprentissage qui permettent aux apprenants de langues d'atteindre les objectifs d'apprentissage. Cet article tente à élaborer un modèle d'apprentissage intégratif qui réunit les quatre compétences langagières.

\section{Discussion}

\subsection{La question d'autonomie d'apprenant}

Par le terme autonome qui dérive du grec (auto-, 'propres' et nomos, 'règles) nous entendons une action exécutée selon leurs propres règles. Autrement-dit, une personne autonome est celle qui est capable ou qui possède la capacité de se contrôler, y compris en déterminant le but, la direction et les limites souhaitées ou nécessaires pour faire quelque chose (Robert, 2002). Dans un contexte de l'apprentissage des langues, une personne peut être qualifiée d'autonome si celle-ci serait capable de gérer de diverses situations de communication avec toutes ses connaissances et ses compétences de la langue cible. Cela signifie qu'il peut déterminer le but, la meilleure direction et les meilleures façons de réaliser l'objectif ultime de l'apprentissage de langue. Ainsi, dans ce cas, la discussion sur l'autonomie ne peut être dissociée du niveau d'apprentissage de la langue. D'ailleurs, l'autonomie d'apprentissage n'est pas convenable pour les débutants qui ne connaissent pas les potentiels sur eux-mêmes. Certes, l'enseignant reste toujours comme une source importante car celui-ci connaît mieux les manières pour déterminer la direction de l'apprentissage en suivant le curriculum et des méthodes systématiques et pertinentes pour un apprentissage des langues.

La position et le rôle de la langue cible dans la vie réelle sont également un facteur inhérent à l'autonomie. La proximité de la langue cible dans la communication sociale pour les apprenants donne une exposition plus ou moins suffisante au développement de stratégies d'apprentissage et réduit la pression mentale et intellectuelle dans le processus d'apprentissage. Le développement de stratégies d'apprentissage tant à l'intérieur qu'à l'extérieur de la classe n'est pas uniquement déterminé par l'interaction des enseignants et des apprenants, mais aussi par l'environnement immédiat qui leur permet d'établir une communication interactive. Par exemple, les gens qui vivent dans une zone fréquentée par des touristes étrangers seront plus largement exposées à la langue cible que celles qui ne le sont pas. De même, ceux qui vivent dans un pays où la langue cible est une langue seconde auront plus de possibilités d'utiliser la langue cible quotidiennement que ceux qui apprennent la langue cible comme langue étrangère.

Dans ce contexte, il y a trois facteurs principaux impliqués dans l'apprentissage et l'acquisition de langues étrangères, en premier lieu, les apprenants de langues étrangères ; deuxièmement, des 
enseignants de langues étrangères ; troisièmement, la langue cible. Les trois facteurs sont liés et deviennent le point de départ de la discussion sur l'autonomie de l'apprentissage des langues étrangères.

Cet article tente de présenter les trois facteurs ci-dessus dans le thème du modèle d'apprentissage intégratif du français effectué dans de différents des quatre compétences langagières. Ce problème est présenté dans le cadre d'une étude de cas sur le modèle d'autonomie de l'apprentissage du français dans la faculté de langues et des arts de l'Universitas Negeri Yogyakarta. Dans cette section, les questions de l'autonomie seront premièrement abordées sous trois angles différents, du point de vue de l'enseignant, du point de vue de l'apprenant et du point de vue du Fle comme la langue cible.

a. Perspective de l'enseignant du Fle (Français langue étrangère)

En général, l'apprentissage du français en Indonésie a été pris en compte dans les nombreuses mises à jour du curriculum et des approches d'apprentissage énoncées dans le curriculum indonésien du cadre de qualifications (RISTEK DIKTI, DOK 1 KKNI, 2015). Le programme KKNI souligne clairement l'importance des qualifications requises et des objectifs d'apprentissage pour que le programme puisse être planifié et mise en place correctement. KKNI fournit une orientation pour l'apprentissage de la langue, qui est ensuite accommodé et systématiquement décrit à travers de certains niveaux d'apprentissage.

En outre, plusieurs accords concernant les acquis de l'apprentissage du français ont déjà été conclus par le biais d'un forum de 10 grandes universités qui enseignent la littérature française et l'enseignement du français en Indonésie. L'accord prévoyait la détermination du seuil pour atteindre au niveau minimum de maîtrise de la langue à la suite du CECRL (Cadre européen de référence pour les langues) en tant que référence pour l'apprentissage du français en Indonésie audelà de l'année 2004.

b. Perspective de l'apprenant du français langue étrangère (FLE)

Les apprenants du français à l'UNY ne commencent pas tous l'apprentissage du français au lycée (faux débutant), la plupart (près de 70\%) ne l'ont jamais étudié et qu'ils sont vrai-débutant.

Les résultats de l'enquête menée en 2016 sur 40 nouveaux étudiants ont montré que 24,6\% des élèves avaient étudié le français au lycée, mais ils ne sont pas au niveau A1.2 CECRL et seulement $5,3 \%$ avaient suivi des cours supplémentaires. Par conséquent, les apprenants du français à l'UNY espèrent que l'apprentissage commence du niveau de base que l'on les considère comme des débutants. En tant que vrai-débutants, les étudiants du premier et deuxième semestre bénéficient de services d'apprentissage entièrement issues de l'enseignant et des manuels.

Le manuel est la source principale et les enseignants fournissent les connaissances de base de la grammaire, de la prononciation et du vocabulaire en tant que modalité d'apprentissage. Les étudiants dépendent presque entièrement de la dynamique d'enseignement et des interactions dans l'apprentissage, notamment en ce qui concerne de l'acquisition de compétences langagières (parler, écouter, lire, écrire).

On peut dire que les apprenants du français ont un outil d'étalonnage qui leur permet de se positionner et de connaitre leur propre situation après un certain moment d'apprentissage. L'étalonnage dans les manuels ECHO se trouve dans chaque unité d'apprentissage avec un système d'auto-évaluation. Considérant que le matériel didactique est basé sur le besoin immédiat en donnant progressivement à l'apprenant un rôle qui mène à une action linguistique, de sorte que les élèves acquièrent progressivement des compétences en interagissant avec les enseignants et leurs camarades. Aux semestres 3 et 4, les élèves devraient être au niveau A2 et devraient immédiatement passer au niveau de préparation à l'autonomie (niveau seuil).

c. Perspective du français langue étrangère parmi d'autres langues vivantes

En Indonésie, le français est l'une des langues étrangères étudiées dans plusieurs grandes villes. Le français n'a pas historiquement ancré au peuple indonésien, soit par la colonisation, soit pas par le lien culturel. De sorte que son existence est un excès ou un résultat du développement de la technologie, du commerce, de l'industrie et de la mondialisation dans le monde. Le français se trouve, parmi d'autres langues étrangères qui coexistent, dans une situation qui ne fournissent pas un environnement propice à l'acquisition de la langue pour ses locuteurs débutants. Cependant, les apprenants qui vivent dans des zones touristiques et des lieux proches du centre de la culture française bénéficient de la possibilité dans un contexte limité de rencontrer et faire du contact avec des locuteurs natifs. Cela signifie que les apprenants qui souhaitent développer leurs compétences linguistiques doivent prendre en compte cette situation, car ce sera un facteur important dans le processus d'acquisition de la langue, de s'exposer au français. On peut dire que la position du français langue étrangère est directement liée à la possibilité pour les apprenants d'être exposés au français. Plus il y a des francophones dans une ville / un lieu, plus il y a des chances d'entendre les 
sons, de pratiquer à parler ou d'utiliser le français dans la communication, d'écouter des nouvelles, de lire des textes, etc.

Ce qui est affirmé précédemment que le discours sur l'autonomie a une dimension très large et est fortement lié aux opportunités chez les apprenants pour développer leurs compétences en français. Le développement de compétences autonome facilité par le milieu constitue une partie importante de l'élaboration des stratégies d'apprentissage du français.

Le problème des stratégies d'apprentissage des élèves est désormais devenu plus complexe qu'un simple environnement physiquement présent autour de l'apprenant. Les apprenants en langues à l'ère de la technologie de l'information vivent dans une époque qui leur permet d'accéder rapidement à l'information à un coût abordable grâce à l'internet et à la facilité d'accès aux medias sociaux.

À cette époque, les stratégies métacognitives joue un rôle indispensable en utilisant la technologie pour appuyer l'apprentissage en raison des ressources d'apprentissage abondantes et de l'accès facile et rapide au multimédia, presque illimitée dans l'espace et dans le temps. Le solide réseau d'accès et de stockage des appareils offre à tout le monde la possibilité de s'adapter à de diverses connaissances et compétences, y compris des compétences langagières. L'apprentissage supporté par des technologies et des stratégies d'apprentissage de langue devient importante et cruciale.

Les stratégies d'apprentissage de langues et l'autonomie d'apprentissage de langues sont deux choses différentes mais portent une relation très étroite. Utiliser l'environnement / le lieu, la technologie pour la réussite de l'apprentissage fait partie de cet effort réalisé à la fois par les apprenants et par les enseignants en langues. Si les apprenants peuvent se libérer peu à peu de la dépendance vis-à-vis de l'enseignant sur les connaissances et les conditions d'apprentissage qu'ils souhaitent, le contrôle de l'apprentissage et de l'acquisition de la langue passera entre les mains des apprenants. Le temps et le lieu ne limitent plus l'interconnexion des apprenants avec les ressources d'apprentissage. Mais d'un autre côté, les rôles et responsabilités des enseignants de langues ont été mis au défi en termes de faciliter l'apprentissage des langues étrangères d'une manière plus subtile.

L'élaboration des stratégies d'apprentissage favorisant l'autonomie de l'apprenant ne libère pas nécessairement la responsabilité et le rôle de l'enseignant de langue. En fait, c'est la capacité de l'enseignant à faciliter le processus d'apprentissage qui se développera. Fukuda, Sakata et Takeuchi (2011) a tenté d'appliquer la procédure d'apprentissage GAS (Guided Autonomy Sylabus) dans une expérimentation visant à améliorer la motivation de l'apprentissage des langues au Japon. Il a constaté que la motivation intrinsèque augmenterait si l'apprentissage plaçait l'importance des relations réciproques entre apprenants - les professeurs d'anglais. Dans cette recherche, il semble que ce que fait l'enseignant a toujours un impact positif sur le processus canalisé par le GAS. Liu (2012), dans le but de concevoir la relation entre les enseignants et les apprenants, examine la relation entre les niveaux d'anxiété, d'autonomie et de motivation pour les langues étrangères et les résultats d'apprentissage. Il a constaté que les niveaux d'anxiété et l'autonomie de l'apprenant contribuaient de manière significative à la prédiction de la réussite des apprentissages en langues étrangères à Taiwan. Sarafianou et Gavriilidou (2015) ont constaté que les interventions d'apprentissage programmées exercés par des enseignants dans l'enseignement de l'anglais langue étrangère a montré une amélioration significative de la stratégie individuelle et collective et sert de fait que la stratégie implicite et intégrée a un rôle positif d'améliorer l'autonomie de l'apprenant.

La réalité est que les questions fondamentales relatives à l'acquisition de compétences linguistiques sont toujours liées à ce que fait l'enseignant et l'apprenant. On suppose que ce problème découle de la fausseté du concept d'enseignement des langues (matériel d'apprentissage moins avancé), des méthodes d'enseignement moins développées, du retard dans l'enseignement, des infrastructures d'enseignement moins modernes. D'autre part, il y a des apprenants avec toutes leurs dispositions et caractéristiques qui deviennent également un facteur distinct. Alors que du côté des enseignants, le problème du faible niveau d'acquisition des compétences linguistiques est toujours orienté vers l'amélioration de la capacité d'enseigner en classe, et non sur la capacité à faciliter (en tant que facilitateur) un groupe d'apprentissage et la facilitation d'un programme autonome. En fait, reconnaître que le rôle d'un enseignant de la langue a évolué au fil du temps et $\mathrm{du}$ développement technologique. Le rôle du facilitateur d'apprentissage ne remplace pas l'enseignement, mais d'ajouter les compétences d'un enseignant de langue. La capacité de faciliter l'apprentissage devient une compétence importante du 21ème siècle, à mesure que les apprenants deviennent plus exposés au monde extérieur. 


\subsection{Faciliter des stratégies dans l'apprentissage intégratif de langue}

\subsubsection{Conditions initiales et processus d'apprentissage}

L'apprentissage intégratif s'est déroulé à six reprises dans le cadre de trois cours de compétences linguistiques, notamment dans le cadre du cours du semestre V de la Compréhension Orale ; l'Expression Ecrite; et le cours de l'Expression Orale. La détermination de ce cours repose sur l'importance de l'expérience de ce modèle en hiérarchisant le problème de l'autonomie de l'apprenant. On suppose que les étudiants de français du 5ème semestre ont commencé à entrer en une phase d'indépendance par rapport à leurs camarades du troisième semestre. L'autonomie est en matière d'apprentissage de la langue, qui correspond au niveau du Cadre européen de référence (CECR). C'est dans cette partie que l'on élabore le sujet de l'autonomie des apprenants dans plusieurs catégories.

1. Disponibilité des apprenants et stratégie métacognitive

D'après le questionnaire remis aux étudiants, on sait que moins de la moitié $(36 \%)$ du nombre total d'élèves participant aux trois cours ne font que parfois planifier l'apprentissage. On peut dire que certains apprenants réfléchissent à l'importance des objectifs, de la planification, des progrès et de l'apprentissage de la langue, alors que les autres n'y pensent peut-être pas trop. Les activités et comportements prévus sont délibérément réalisés avec différentes intensités et variations. Certains apprenants n'attendent même que les instructions proposées par l'enseignant plutôt que de penser à ce qu'ils peuvent créer. Ils croient que les programmes d'apprentissage des langues offerts par apprentissage intégratif leur ont été bien planifiés et qu'ils n'ont pas à faire trop d'efforts pour apprendre les langues.

Il semble que les étudiants sont habitués en fonction du programme fourni par l'enseignant. Les données obtenues montrent que certains apprenants manquent d'indépendance, ce qui signifie qu'ils ne commencent pas immédiatement à apprendre avant de recevoir un stimulus ou une instruction. Certains indicateurs présentent ce phénomène. $46 \%$ des répondants pensent à l'organisation de l'apprentissage. 55\% des répondants sont capables de faire une auto-évaluation. Cela peut s'expliquer par l'intensité de la formation dispensée et les tests qui encouragent les étudiants à se préparer à l'examen afin de ne pas avoir de mauvaises notes au cours.

2. Stratégie cognitive dans le processus d'apprentissage Intégratif

Plus de la moitié des étudiants ayant suivi de l'apprentissage intégratif (65\%) interrogés ont déclaré qu'ils préfèrent utiliser ou appliquer de diverses techniques à mémoriser / mnémonique face aux médias et au matériel pédagogique.

Cette technique est un comportement qui rassemble plusieurs autres stratégies d'apprentissage telles que la répétition, la révision, la vérification des mots dans le dictionnaire, la prise de notes, la création de cartes conceptuelles, etc., dont le but est de retenir les connaissances. Dans ce cas, les étudiants appliquent plusieurs stratégies pour retenir des matériels d'apprentissage du français tels que le vocabulaire, les règles de la grammaire, les règles de la prononciation, etc. Selon certains étudiants, certaines activités de mémorisation telles que la répétition, la révision, la prise de notes pour de nouvelles connaissances ou des leçons leur donnent de bons résultats. Ainsi, certaines techniques d'apprentissage telles que le remue-méninge, le groupement de mots, la répétition, la révision sont un ensemble de techniques incluses dans les stratégies d'apprentissage privilégiées par les étudiants pour mémoriser de nouvelles matières qui ne sont en réalité que des stratégies cognitives. Nous pouvons établir un lien entre ce phénomène et les situations d'apprentissage où les élèves attendent et enregistrent ce qui est enseigné et expliqué par l'enseignant dans le cas des cours conventionnels, sans avoir à se préoccuper de l'autoévaluation. En fait, les apprenants peuvent faire quelque chose pour ne pas rester silencieux et passif au cours d'apprentissage. On peut dire que la réticence de ces étudiants peut influencer les stratégies sociales pour comprendre et reconnaître de nouvelles compétences. Les caractéristiques de ces étudiants ont également été exprimées dans le récit des élèves interrogés.

D'autres techniques considérées comme des stratégies cognitives sont la traduction et la paraphrase. Ces stratégies sont utilisées par plusieurs étudiants $(27 \%$ et $55 \%)$ qui exigent apprendre avec ces deux techniques. La technique de traduction est utilisée en raison que les élèves peuvent comparer les règles de la phrase qu'ils ont connue avec ce qu'ils apprendront. Ils utilisent un mot ou une phrase dans une autre langue pour répondre à la question de l'enseignant. Ils ne remettent pas en question le manque de vocabulaire en changeant de code s'ils rencontrent certaines difficultés. Ils peuvent choisir d'autres mots, avec d'autres explications, ou s'ils sont 
obligés d'utiliser le langage des signes pour des raisons de communication. Ces méthodes peuvent être considérées comme des techniques dans les stratégies compensatoires. Cela se produit en raison de divers problèmes généralement rencontrés par les apprenants dont la compétence de communication est encore faible face à la complexité du français.

La priorité de l'apprentissage des langues est la capacité de développer des moyens efficaces d'exprimer leurs pensées et leurs idées. La relation avec les stratégies cognitives est que, dans l'apprentissage intégratif, ces moyens conventionnels d'utilisation peuvent encore être utilisés et d'une manière naturelle par les individus pour résoudre les problèmes de langage et de communication. Des habitudes rendront la langue cible plus facile à mémoriser et la rendre automatiquement car il se fait d'une manière répétitive.

3. Apprentissage intégratif et conditions socio-affectives (peur, honte, réticence)

Le problème de la condition socio-affective des apprenants est la question de l'intensité avec laquelle l'apprenant profite de la possibilité d'interagir en français pour pratiquer la langue qu'il apprend. De nombreux problèmes peuvent empêcher un apprenant de la langue de prendre parti de la possibilité de pratiquer la langue cible. Parmi ceux-ci, il y a la peur ou la honte qui est affichée en public. Dans les cours de langue, la situation ne devrait pas décourager les étudiants à utiliser la langue cible, mais les apprenants doivent se sentir libres d'exprimer ce qu'ils savent de la langue qu'ils apprennent. Dans les cours de français, les élèves ne divulguent pas toujours facilement ce qu'ils veulent demander ou confirmer avec l'enseignant. Les raisons peuvent être diverses, mais que ce soit dans un environnement, elles devraient pouvoir de faire des erreurs, elles n'ont pas de prix.

En ce qui concerne des méthodes mises en œuvre par les apprenants pour surmonter les difficultés d'apprentissage, il s'avère que l'absence de matériel de référence est l'une des raisons pour lesquelles la langue cible n'est pas utilisée. Les élèves développent une variété de techniques, y compris des stratégies métacognitives, en se référant à tous les ouvrages qu'ils veulent obtenir comme des réponses à leur ignorance. Les références en question peuvent être des encyclopédies en ligne, des dictionnaires en ligne, des grammaires, des cahiers et d'autres références dans leurs appareils. À l'inverse, avec un accès facile à Internet, le besoin d'interaction avec les enseignants n'est plus fonctionnel. Les élèves comptent davantage sur Internet pour trouver des informations que de demander à l'enseignant. Cela peut se produire si la raison en est la satisfaction des réponses obtenues, car après tout, le réseau Internet fournit une variété d'informations presque illimitées. Mais la nécessité d'interagir dans cette classe de langue ne repose pas uniquement sur les intérêts du contenu de l'information, mais également sur la possibilité d'utiliser ou de pratiquer la langue cible de manière interactive. Par exemple, si les élèves veulent communiquer verbalement, mais que leur interlocuteur ne le comprend pas, il y aura un processus d'apprentissage de la prononciation qui donne un effet sur la rétention des règles phonologiques.

On peut donc dire que les apprenants qui utilisent la possibilité de communiquer dans le cadre d'un apprentissage intégratif utilisent inconsciemment des stratégies sociales passant par le contact avec leurs interlocuteurs ou leur environnement. Ils utilisent également des stratégies affectives en réduisant la peur ou le doute lors de la prise de parole. Un autre fait concernant le courage de prise de parole est que seulement $15 \%$ des étudiants interrogées sont conscient qu'ils utilisent l'occasion et établissent en effet des contacts tout en cherchant des informations ou des explications. Les autres font la même chose mais ne se rendent pas compte qu'ils mettent en place des stratégies d'apprentissage socioaffectif. D'autres problèmes sont toujours liés à ce qui précède, à savoir le contrôle émotionnel ou la maîtrise de soi. $38 \%$ des personnes interrogées font toujours preuve de maîtrise de soi avant de faire des gestes linguistiques, en particulier pour ne pas être nerveuses. La technique peut varier, par exemple en prenant une profonde respiration.

On peut dire que les apprenants qui profitent d'opportunités d'expression telles que parler, discuter en se tenant debout devant leurs amis dans des cours sont ceux qui contrôlent leurs émotions. Pourquoi certains peuvent-ils le faire alors que d'autres ne le font pas? Il est intéressant de savoir comment un élève peut surmonter sa peur et son inquiétude pour saisir une opportunité. Les étudiants ne veulent pas que la peur bloque leurs démarches pour exprimer et écouter les opinions des autres en langue cible. Le fait est que les étudiants font les deux stratégies en même temps.

La première stratégie est la planification du discours (st. Métacognitive), puis la prise de contrôle sur son émotion (st Affective), puis l'acte d'exécution et la sélection des codes linguistiques. La prise de parole dans son propre environnement est une forme des stratégies sociales dans l'apprentissage des langues. En outre, le contrôle affectif est une stratégie socioaffective qui permet aux apprenants de la langue de rompre avec les limites du doute, de la 
peur et de la honte qui ne favorisent pas l'apprentissage des langues. Dans beaucoup d'études menées par des didacticiens des langues, on a trouvé une corrélation positive entre la honte, la peur et la motivation à apprendre (Gardner, Lambert, 1972). Si tel est le cas, on peut dire que la stratégie menée par les étudiants est la solution personnelle pour surmonter la détresse psychologique.

Le contact social et la collaboration sont deux choses presque identiques. La compatibilité qui existe entre l'individu et l'environnement permet aux apprenants d'interagir et de coopérer avec son environnement. Mais cela reste difficile à faire pour beaucoup d'apprenants en raison des barrières psychologiques. La honte et la peur élimine lentement la confiance en soi car elle se déplace vers une situation plus facile. Faciliter un espace pour surfer dans le cyberespace rend les élèves deviennent des apprenants riches en informations mais ne les rends pas habitués à la coopération. Démontré par les données du questionnaire sur la coopération, ce phénomène ne représente que $23 \%$ du total des répondants. La coopération dans la résolution des problèmes d'apprentissage de l'écoute et la compréhension en français est quelque chose d'habituel pour tout apprenant, en particulier pour ce qui concerne l'amélioration des compétences de communication en français.

Un autre aspect de l'apprentissage intégratif est le problème socioaffectif en ce qui concerne l'auto-renforcement. De nombreux apprenants ont le sentiment qu'ils doivent lutter pour maîtriser le matériel d'apprentissage sans se rendre compte que cela n'est possible que si le matériel précédent est correctement acquis. Si le processus d'acquisition de la langue se déroule bien, certaines parties de la langue cible ont été internalisées et sont devenues automatiques chez l'apprenant.

Mais ce processus doit passer par un processus de renforcement qui peut ressembler à des exercices grammaticaux réalisé en continu. Quel que soit le but des compétences linguistiques à atteindre, il faut une pratique répétée. S'exprimer peut-être plus facile si l'on a l'habitude de parler, d'écrire, d'écouter et de lire. Malheureusement, cela ne se fait que par peu d'apprenants en se mettant dans un environnement approprié (lire des livres, des bandes dessinées, des magazines ou regarder des films, la télévision ou écouter des documents audio ou radio français).

À partir du questionnaire, 52\% des répondants souhaitaient avoir un ami francophone pour pouvoir interagir en français. Alors que $50 \%$ ont déclaré être suffisamment ouverts pour partager les uns avec les autres lorsqu'ils éprouvaient des difficultés à apprendre le français. Dans ce cas, $10 \%$ ont admis qu'ils n'osaient pas écrire de statut sur les réseaux sociaux ou parler en français.

Il est important de poursuivre cet apprentissage intégratif en ce qui concerne l'apprentissage des matériels et des stratégies. Premièrement, il faut confirmer aux élèves dans le processus de précondition à propos de la difficulté à laquelle ils font face en ce qui concerne la compréhension, la prononciation et la prise de parole. La prononciation française reste difficile pour locuteur de la langue indonésienne, mais les réponses des élèves mèneront l'apprentissage intégratif vers des activités créatives comportant certaines stratégies.

Face à ce problème, tout d'abord les apprenants doivent être capable de mettre en place des stratégies métacognitives (par exemples pour vérifier des questions en prenant des notes, consulter le site web); des stratégies cognitives (en imitant, en périphrasant des mots difficiles);et des stratégies affectives (en établissant autant de communication que possible en français avec les élèves). Deuxièmement il faut confirmer aux élèves ce qu'ils pensent de meilleures stratégies pour eux. Les trois types de stratégies qu'ils utilisent sont différents, mais ils seront toujours très utiles, par exemple, X préfère étudier d'une façon métacognitive en se dotant de nombreux vocabulaire et de connaissances grammaticales. L'Y utilise une stratégie cognitive qui consiste à analyser et à faire des conclusions déductives et inductives pour sa compréhension. Et le Z utilise une stratégie socioaffective, en prenant autant du contact avec d'autres personnes en langue cible, en particulier avec des locuteurs natifs et des enseignants. La troisième solution concerne les compétences en écriture et la maîtrise de la grammaire. Les étudiants considèrent généralement que la grammaire dispose de certains types de phrase de sorte qu'ils les principalement mémorisent. Pour écrire, ils s'appuient fortement sur la capacité de passer ces types par la maîtrise du vocabulaire. Ainsi, toutes les questions liées à l'écriture seront également liées à la grammaire qu'elles maîtrisent. On peut en comprendre que l'apprentissage écrit met l'accent sur les aspects cognitifs plutôt que sur les aspects sociaux. La plupart des étudiants ne sont pas habitués à écrire des textes sur ses médias sociaux en français avant de savoir à l'avance que la phrase à écrire est correcte grammaticalement. Cependant, certains étudiants ont déclaré qu'ils discutaient souvent par écrit dans le cyberespace en français avec des locuteurs natifs. Ce comportement est très intéressant car il révèle que l'apprenant ne veut pas que toutes leurs défauts soient exposés dans la conversation 
et jugées parles autres. Avec des locuteurs natifs qu'ils ne connaissent pas, ils peuvent cacher leur identité et se comporter en toute sécurité sans avoir à supporter la honte d'erreurs ou de fautes. L'intelligibilité mutuelle ou la compréhension en communication constitue le but que les étudiants veulent atteindre.

\section{Conclusion}

On peut en conclure que l'apprentissage intégratif permettra aux étudiants d'avoir les compétences linguistiques d'une façon plus proportionnelle où les enseignants et les apprenants sont libres d'établir le contact avec la langue cible passant par les stratégies d'apprentissage préférées par les apprenants. L'autonomie sera une question d'apprivoiser les difficultés ou bien de les surmonter avec leur propre moyen.

Ce modèle implique une conception d'apprentissage qui préoccupe l'importance d'opportunités disposé aux apprenants dans le processus d'apprentissage de la langue. L'opportunité offerte par le biais d'un processus d'immersion établit un environnement disponible à l'autonomie d'apprentissage. Les relations d'enseignant et d'apprenant dans tous leurs sens ne doivent pas nuire à l'existence de ces opportunités et, au contraire, il faudra renforcer les bonnes stratégies d'apprentissage.

La formation sur les stratégies d'apprentissage des langues peut être possible à faire, par exemple en fournissant un traitement qui stimule les apprenants faibles à développer un comportement adaptatif aux environnements d'apprentissage qui leur donnent une chance. Favoriser des attitudes positives à l'égard de ces comportements permettant d'associer les relations sociales et interpersonnelles à l'autonomie de l'apprenant.

\section{Références}

Chamot, A. U. (2004). Issues in Language Learning Strategy Research and Teaching. Electronic Journal of Foreign Language Teaching, 1(1), 14-26. Retrieved from http://e-flt.nus.edu.sg/v1n12004/chamot.pdf

Chamot, A. U., \& Kupper, L. (1989). Learning Strategies in Foreign Language Instruction. Foreign Language Annals, 22(1), 13-22. https://doi.org/10.1111/j.1944-9720.1989.tb03138.x

Fukuda, S., Sakata, H., \& Takeuchi, M. (2011). Facilitating autonomy to enhance motivation: Examining the effects of a guided-autonomy syllabus. Electronic Journal of Foreign Language Teaching, 8(1), 71-86.

O’Malley, J. M., \& Chamot, A. U. (1990). Learning Strategies in Second Language Acquisition. Cambridge:

Cambridge University Press. https://doi.org/10.2307/415153

Oxford, R. L. (2003). Toward a More Systematic Model of L2 Learner Autonomy. In D. Palfreyman \& R. C. Smith (Eds.), Learner Autonomy across Cultures (pp. 75-91). Hampshire : Pa. https://doi.org/10.1057/9780230504684_5

Robert, J.-P. (2002). Dictionnaire pratique de didactique du FLE. Paris: Editions OPHRYS.

Rubin, J. (1975). What the “Good Language Learner” Can Teach Us. TESOL Quarterly, 9(1), 41-51. https://doi.org/10.2307/3586011 